\title{
Surface adhesion of back-illuminated ultrafast laser-treated polymers
}

\author{
Deepak L. N. Kallepalli $\odot,{ }^{*}$ Alan T. K. Godfrey, Jesse Ratté $\odot$, André Staudte, Chunmei Zhang, and P. B. Corkum $\odot^{\dagger}$ \\ Joint Attosecond Science Laboratory, University of Ottawa and the National Research Council of Canada, \\ 100 Sussex Dr., Ottawa, Canada K1N 6N5
}

(Received 31 August 2020; revised 16 February 2021; accepted 6 April 2021; published 28 April 2021)

\begin{abstract}
We report a decreased surface wettability when polymer films on a glass substrate are treated by ultrafast laser pulses in a back-illumination geometry. We propose that back illumination through the substrate confines chemical changes beneath the surface of polymer films, leaving the surface blistered but chemically intact. To confirm this hypothesis, we measure the phase contrast of the polymer when imaged with a focused ion beam. We observe a void at the polymer-quartz interface that results from the expansion of an ultrafast laser-induced plasma. A modified polymer layer surrounds the void, but otherwise the film seems unmodified. We also use x-ray photoelectron spectroscopy to confirm that there is no chemical change to the surface. When patterned with partially overlapping blisters, our polymer surface shows increased hydrophobicity. The increased hydrophobicity of back-illuminated surfaces can only result from the morphological change. This contrasts with the combined chemical and morphological changes of the polymer surface caused by a front-illumination geometry.
\end{abstract}

DOI: 10.1103/PhysRevMaterials.5.045201

\section{INTRODUCTION}

The use of ultrafast pulses for local energy deposition in materials has several applications in 3D optical data storage $[1,2]$, integrated optics [3-5], and laser-induced forward transfer (LIFT) [6-11]. In contaminant-free LIFT, the nonlinear interaction of an ultrafast pulse with the polymer film (often called a dynamic release layer) between a glass substrate and transfer material ensures the confinement of the energy deposition to the glass-polymer interface, leaving the transfer material chemically intact [12]. When an ultrafast femtosecond pulse interacts with a polymer film at the interface, it creates a localized hot plasma $[13,14]$. The hot plasma expands, leading to formation of a blister.

The adhesive properties of materials are altered by both front and back illumination. However, front illumination induces both morphological and chemical changes to the surface $[15,16]$. Front-illuminated surfaces can lead to hydrophilic, hydrophobic, and superhydrophobic states in addition to chemical changes [17-25]. Thus, changes to surface adhesion are often attributed to an interplay between surface chemistry and morphology, in particular for front-illuminated surfaces. To the best of our knowledge there have been no reports of changes to adhesion induced by an ultrafast laser in a backilluminated geometry.

We report an increase in hydrophobicity of a polyimide film induced by an ultrafast laser in a back-illumination geometry. Using focused ion beam (FIB) microscope and $\mathrm{X}$-ray photoelectron spectroscopy (XPS), we find that these changes are strictly morphological at the interface of the film. This observation is consistent with our previous spectroscopic

\footnotetext{
*Deepak.Kallepalli@uottawa.ca

†pcorkum@uottawa.ca
}

measurements, which showed that laser-induced chemical changes are confined to the glass-polyimide interface [12]. We confirm the increased hydrophobicity by measuring the contact angle of water droplets on laser-modified surfaces, and we find that the contact angle increases with laser fluence.

\section{EXPERIMENT}

We prepared polyimide films on two different substrates: (1) on \#1.5 Fisherbrand borosilicate glass coverslips (for FIB and contact angle measurements) and (2) on $500 \mu \mathrm{m}$ fused silica discs for our XPS measurements. Both were rinsed with acetone, isopropanol, and deionized water to remove contaminants and dried on a hotplate. Polyimide films were made using PI-2525 (for thicker films) and PI-2555 (for thinner films $\leqslant 1.4 \mu \mathrm{m}$ ) precursors from HD Microsystems following the recommended spin curves and baking conditions.

We used a Ti:Sapphire laser (Coherent RegA 9040) producing pulses of 50-fs duration at a central wavelength of $800 \mathrm{~nm}$. The laser pulse energy was controlled using a motorized half-wave plate followed by a polarizing beam-splitter cube. The laser beam was focused using microscope objectives $(10 \times 0.2 \mathrm{NA}, 20 \times 0.4 \mathrm{NA})$ mounted into a vertical motor stage (PI M-112) with a travel range of $25 \mathrm{~mm}$ for adjusting the focal spot on the sample. Since polyimide is transparent at a wavelength of $800 \mathrm{~nm}(1.55 \mathrm{eV})$, any modification is due to nonlinear absorption (Supplemental Material Sec. I [26]).

We mounted polyimide-on-glass coverslips onto a five-axis piezo nanoprecision stage (PI) assembled on top of a microprecision horizontal XY stage (MICOS MS-4). The nanoprecision stage was used for fine adjustment of the focal position. The laser was focused through the glass substrate onto the glass-polymer interface (back-illumination geometry). A dichroic mirror was used before the microscope objective in a coaxial geometry, allowing a small portion of 
the focused laser light to back-reflect from the sample, recollimate through the objective, and travel to an imaging line for in situ laser spot monitoring. Coupling in white light and changing the position of the objective also allows for in situ white-light microscopy. We used this to find the optimal position of the laser focus on the sample, by firing pulses with energies near the damage threshold while adjusting the position of the focal spot. A schematic of our experimental setup and characterization details are in Supplemental Material Secs. II and III [26].

\section{RESULTS AND DISCUSSION}

The self-focusing threshold in the substrate is $P=2.8 \mathrm{MW}$, and for a 50 -fs pulse this corresponds to $140 \mathrm{~nJ}$ energy. Despite the losses in the substrate, the remaining energy delivered to the polymer film at the interface leads to a blister formation. Increasing the energy, especially for a low NA lens, will lead to continuum generation and breakdown in the substrate, in addition to blister formation [13]. Here, we accounted for the substrate-mediated absorption in glass using the lawn-mower model published in our earlier work $[13,14]$. In this section, we report pulse energies delivered through the substrate accounting for the nonlinear absorption in the substrate. The minimum number of photons required for modification in polymer is 2 (see absorption spectrum of pristine polymer in Supplemental Material Sec. I [26]).

We carried out laser irradiation experiments using thin polyimide films on glass and examined the changes in chemical composition and morphology at the glass-polymer interface and the surface. Contaminant-free LIFT requires confinement of chemical changes near the glass-polyimide interface while the polymer surface undergoes a morphological change through blister formation. Since polymers possess lower surface energies ( $\sim 40 \mathrm{~mJ} / \mathrm{m}^{2}$ for polyimide) compared to metals $\left(\sim \mathrm{J} / \mathrm{m}^{2}\right)$, we chose a test polymer and carried out studies on chemical composition and morphology [27-29]. We used FIB and XPS measurements to study the morphology and chemical composition. We show hydrophobicity test results on laser-irradiated polyimide surfaces to demonstrate its relevance to the LIFT technique and other applications.

\section{A. Chemical composition of the surface}

In this section, we present results from FIB measurements that image changes near the interface, followed by XPS measurements. For FIB analysis, we fabricated a series of individual blisters with $\approx 130 \mathrm{~nJ}$ of pulse energy focused by a 0.4 NA microscope objective reaching an intensity of $5.3 \times$ $10^{13} \mathrm{~W} / \mathrm{cm}^{2}$ at the interface. All laser-irradiation experiments were carried out by focusing pulses at the glass-polyimide interface.

We used focused gallium and helium ion beams to dissect and image the interior of blisters, respectively, as shown in Fig. 1(a). The dissected blister showed a thin embedded layer of modification underneath the unmodified polymer in the blister. The image contrast indicates that only a very thin layer on the underside of the blister has undergone chemical transformation. In addition, a void is present beneath the
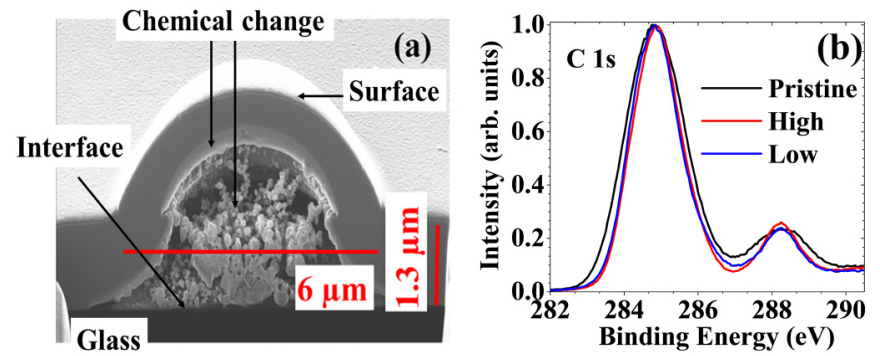

FIG. 1. (a) FIB image of a blister fabricated with $\sim 130 \mathrm{~nJ}$ pulse energy focused by a 0.4 NA objective $\left(\sim 2.8 \mathrm{~J} / \mathrm{cm}^{2}\right)$. The height and diameter of the blister are $1.6 \mu \mathrm{m}$ and $6 \mu \mathrm{m}$, respectively. Glass (substrate), interface, and unmodified surface are indicated with black arrows. The chemical changes are apparent in the different brightness compared to the surrounding material. (b) XPS of the carbon (1s) absorption edge for pristine (black) and laser-modified polyimide (red and blue). The laser-modified polyimide was subjected to a low dose of $\sim 1.8 \mathrm{~J} / \mathrm{cm}^{2}$ (blue curve) and a high dose of $\sim 4.7 \mathrm{~J} / \mathrm{cm}^{2}$ (red curve). Normalized intensity for $284.85 \mathrm{eV}$ peak is plotted on the $\mathrm{Y}$ axis for comparison.

embedded layer. It is partially filled with molten material that has solidified.

This observation is consistent with what we expect. When an ultrafast laser pulse is focused into the material interface, it forms a plasma [13]. At the temperature that we achieve, the material vaporizes and undergoes a chemical change creating the embedded layer and the solidified material on glass, as seen in Fig. 1(a). Earlier reports involving blister formation in thin films of polyimide and titanium using $355 \mathrm{~nm}$ and $800 \mathrm{~nm}$ laser wavelengths lack the direct experimental evidence of confined chemical changes at the interface [5,11,30,31]. Our observation of confined chemical changes provides direct experimental evidence for confinement of chemical changes only at the interface induced by an ultrafast laser [5-8,30-42].

XPS is a surface characterization technique with a typical sampling depth of $7.5 \mathrm{~nm}[43,44]$. Hence, we chose XPS to study any chemical changes that occurred to the front surface in back-illuminated experiments on polyimide. Conventional spectroscopic techniques, such as absorption and fluorescence, are not useful in studying local changes occurring at specific depths as these measurements result in a combined spectrum of pristine polymer surface, modified polymer at the interface, and glass [12].

In our earlier report, we recorded local photoluminescence changes using confocal micro-Raman and fluorescence techniques [12]. We observed photoluminescence changes more confined to the edges than the center of these individual blisters when excited at different wavelengths using a 0.9 NA objective. However, this technique cannot resolve the modified polymer at the interface from the film surface, due to the limited depth resolution (axial resolution of 1.5$2.3 \mu \mathrm{m})$. Therefore, we chose XPS characterization for this study as $\mathrm{x}$ rays offer better depth resolution compared to these techniques. XPS measurements provided isolated information about the chemistry of the film surface.

Since XPS characterization requires a large, patterned surface area, we patterned $2 \mathrm{~mm} \times 3 \mathrm{~mm}$ areas on $1.3 \mu \mathrm{m}$ thin 
films of PI-2555 by overlapping the individual blisters in back-illumination geometry. Two regions were modified with $\sim 4.7 \mathrm{~J} / \mathrm{cm}^{2}$ (high irradiation dose) and $\sim 1.8 \mathrm{~J} / \mathrm{cm}^{2}$ (low irradiation dose). The calculation of energy densities and irradiation details can be found in Supplemental Material Secs. IV and $\mathrm{V}$ [26].

Figure 1(b) shows the carbon (1s) absorption edge for pristine and laser-irradiated samples (black, red, and blue for high and low irradiation dosed polyimide). For pristine polyimide, the carbon absorption edge [black curve in Fig. 1(b)] consists of two peaks at $288.35 \mathrm{eV}$ and $284.85 \mathrm{eV}$ corresponding to $\mathrm{C}=\mathrm{O}$ and $\mathrm{C}-\mathrm{C} / \mathrm{C}-\mathrm{H}$, respectively. The XPS spectra recorded for laser-modified polyimide surfaces [red and blue curves in Fig. 1(b)] were corrected for energy shifts due to charge compensation $(\approx 2 \mathrm{eV})$ and plotted to compare with pristine polyimide [24]. There were no peak shifts and/or new peaks observed, indicating that the surface is chemically intact after the laser treatment. We also compared the intensity ratios of peaks at $284.85 \mathrm{eV}(\mathrm{C}-\mathrm{C} / \mathrm{C}-\mathrm{H})$ and $288.35 \mathrm{eV}(\mathrm{C}=\mathrm{O})$ for laser-modified polyimide samples (blue and red curves) with the pristine polyimide. Since the morphology of the surface is altered after the laser treatment, the resulting number of photoelectrons generated also changes. Hence, intensity ratios of peaks are compared rather than their individual intensities [45]. The normalized intensity ratios of peaks for laser-modified samples were nearly equal when compared with the pristine polyimide (nearly 4). From this, we conclude that the carbon bonds at the surface of film were not altered. We also compared $\mathrm{O}(1 \mathrm{~s})$ and $\mathrm{N}(1 \mathrm{~s})$ envelopes for a laser modified with pristine polyimide and did not observe any peaks shift or any significant changes in the intensity ratios (details are in Supplemental Material Sec. V [26]), further confirming that the surface chemistry was not altered.

\section{B. Role of surface morphology on adhesion}

Having determined that back-illuminated ultrafast lasertreated polymers leave the chemical composition of the polymer intact, we next turn to the role of surface morphology (roughness ratio) on surface adhesion. It is known that surface adhesion is influenced by both chemistry and morphology. When a water droplet is placed on a flat surface (zero roughness), it shows a contact angle known as Young's angle $\left(\Theta_{0}\right)$ illustrated in Fig. 2(a). The contact angle results from a balanced surface force between three interfaces: air-solid, solid-liquid, and liquid-air.

The initial chemical state of a polymer surface depends on the baking conditions (such as temperature or time). When polymers are baked for a long time or at higher temperatures, hydrophilic groups are removed resulting in enhanced hydrophobicity. Figures 2(b) and 2(c) show the contact angles of $110^{\circ}\left( \pm 0.5^{\circ}\right)$ and $82^{\circ}\left( \pm 0.5^{\circ}\right)$ for water droplets placed on polyimide thin films, which were prepared by baking at $300^{\circ} \mathrm{C}$ and $180^{\circ} \mathrm{C}$ for $30 \mathrm{mins}$, respectively.

When surface texture is added to any substrate in back illumination, it increases its initial hydrophilic or hydrophobic state. Since our experiments are carried out in back-illumination geometry on polymers prepared at $300^{\circ} \mathrm{C}$, the addition of surface texture enhances hydrophobicity as shown in Fig. 2(d). In experiments, the surface texture

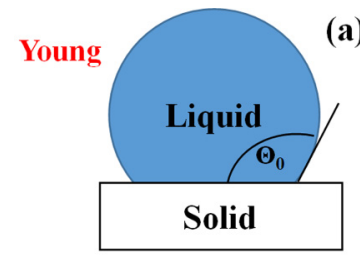

Cassie-Baxter

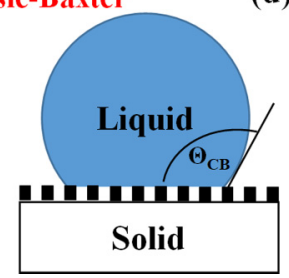

(d)

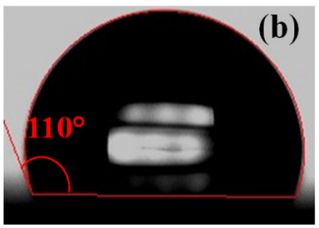

FIG. 2. (a) A flat substrate showing Young's contact angle $\left(\Theta_{0}\right)$. (b) and (c) show contact angles of $110^{\circ}\left( \pm 0.5^{\circ}\right)$ and $82^{\circ}\left( \pm 0.5^{\circ}\right)$ of pristine polyimide films baked at $300^{\circ} \mathrm{C}$ and $180^{\circ} \mathrm{C}$ for 30 mins. (d) Young's contact angle $\left(\Theta_{0}\right)$ transforms to Cassie-Baxter angle $\left(\Theta_{\mathrm{CB}}\right)$ for hydrophobic surfaces upon addition of surface texture. Additional surface texture increases its initial hydrophobicity $\left(\Theta_{\mathrm{CB}}>\Theta_{0}\right)$.

induced by an ultrafast laser is pointlike due to the intensity distribution of the pulse (blisters which are pointlike objects), in contrast to the uniform surface texture (periodically placed pillars), shown in Fig. 2(d).

Since a $1 \mu \mathrm{L}$ water droplet requires a $\sim \mathrm{mm}^{2}$ surface area for a hydrophobicity measurement, we patterned surface areas of $2 \mathrm{~mm} \times 3 \mathrm{~mm}$ under a $10^{-3}$ Torr vacuum (to avoid interaction with atmospheric oxygen [24]). We patterned surfaces with 1, 2, and 4 shots per focal spot diameter on average $(N)$, by varying scan speed $(v)$, laser repetition rate $(L)$, and line spacing $(\Delta X)$ (details in Supplemental Material Sec. VI). AFM topographies of the patterned surfaces are shown in Fig. 3. In AFM related studies, the average surface roughness $(R)$ is defined as the arithmetic mean of the absolute values of the roughness profile ordinates. In our case, the pristine polyimide had an average surface roughness $(R)$ of $0.2 \mathrm{~nm}$ and a surface height of $8 \mathrm{~nm}$. Patterned surfaces with $N=1,2$, and 4 had average roughnesses of $225 \mathrm{~nm}, 125 \mathrm{~nm}$, and $59 \mathrm{~nm}$ with surface heights of $1.2 \mu \mathrm{m}, 800 \mathrm{~nm}$, and $350 \mathrm{~nm}$, respectively. The maximum patterned surface heights for $N=4$ and 2 were less than the maximum heights for $N=1$. The energy (E) used to pattern these surfaces was varied with $N$ to avoid rupture of the film.

If laser-induced surface texture is added to an initially flat surface in back illumination, it changes only the adhesive properties (preserving the surface chemistry) by minimizing the contact area fraction via pointlike blister formation [13]. When large surface areas are patterned using an ultrafast laser, the laser-induced surface texture contains complex rough patterns (in 3D) causing a locally increased hydrophobicity due to an increase in the density of air pockets. Hence, the wettability (measured by the liquid contact area fraction) exhibited by such surfaces depends directly on the nature of the laserinduced surface texture that includes the properties of blisters (diameter, height, line spacing, overlap in scan direction, and the shape). 


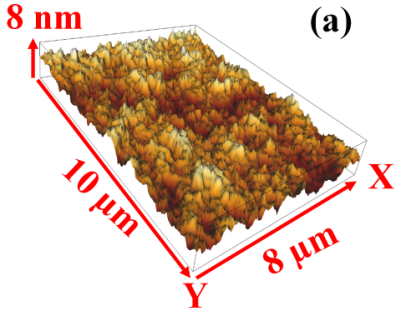

Pristine, $\mathrm{R}=0.2 \mathrm{~nm}$

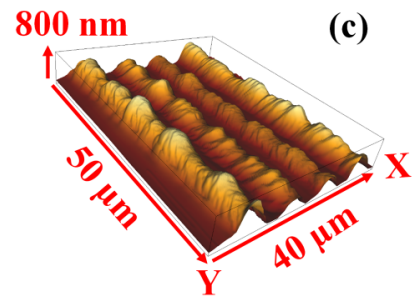

$260 \mathrm{~nJ}, \mathrm{~N}=2, \mathrm{R}=125 \mathrm{~nm}$

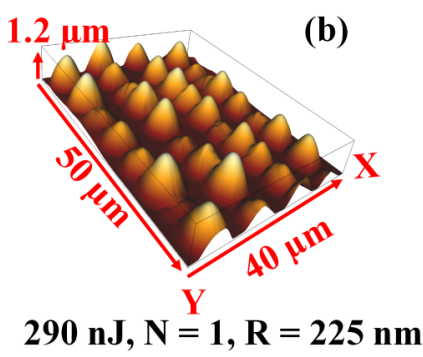

$350 \mathrm{~nm}$

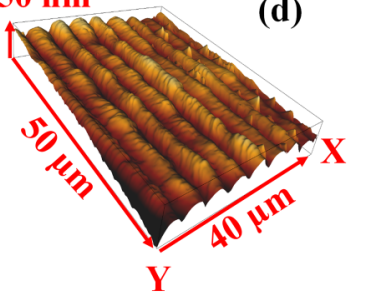

$220 \mathrm{~nJ}, \mathrm{~N}=4, \mathrm{R}=59 \mathrm{~nm}$

FIG. 3. (a) AFM image of a pristine polyimide surface with average roughness of $0.2 \mathrm{~nm}$ with a maximum surface height of $8 \mathrm{~nm}$. Surface topographies shown in (b), (c), and (d) are laser-patterned surfaces of polyimide fabricated with different energies $(E)$, no. of shots $(N)$, average surface roughness $(R)$, at line spacings $(\Delta \mathrm{X})$ of $10 \mu \mathrm{m}, 9 \mu \mathrm{m}$, and $5 \mu \mathrm{m}$, respectively.

The addition of laser-induced surface texture to an existing hydrophobic surface [Fig. 4(a)] in back illumination transforms it into a superhydrophobic surface by minimizing the liquid contact area fraction $(f)$ given by the Cassie-Baxter equation $\cos \left(\Theta_{\mathrm{CB}}\right)=f\left(1+r \cos \left(\Theta_{0}\right)\right)-1$ [46] [equation (17) in Ref. [46]], where $r$ is the ratio of the actual surface to the geometric surface. The liquid contact area fraction $(f)$ is a dimensionless number (unlike the average surface roughness

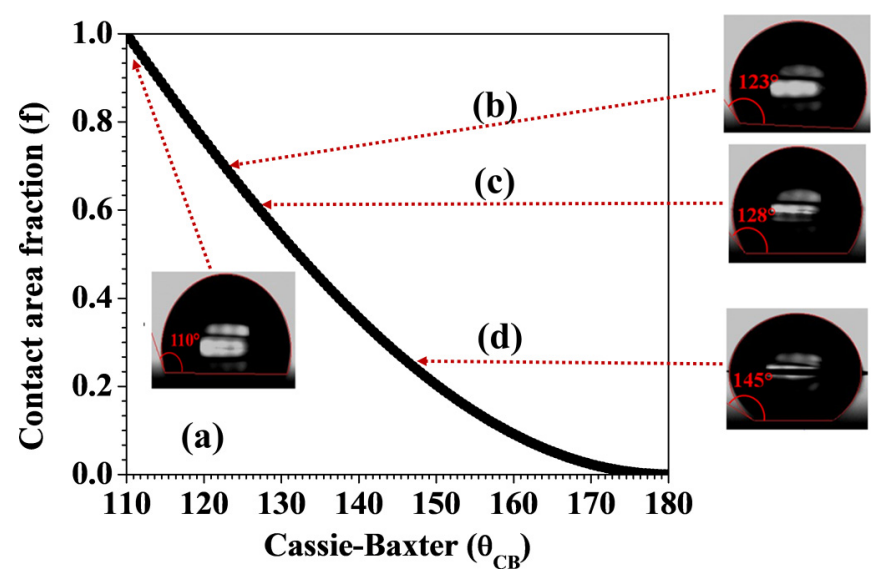

FIG. 4. Plot of liquid contact area fraction $(f)$ dependence on the Cassie-Baxter angle $\left(\Theta_{\mathrm{CB}}\right)$. The curve was obtained under the assumption of our initial polyimide surfaces to be flat. The accuracy of the goniometer was $\pm 0.5^{\circ}$. The acquisition software automatically added the contour shown in red and provided the contact angles. Water contact angle measurement of (a) pristine polyimide $\left(110^{\circ} \pm\right.$ $0.5^{\circ}$ ). Here, both the Cassie-Baxter and Young's contact angle are equal $\left(\Theta_{\mathrm{CB}}=\Theta_{0}\right)$. Laser-patterned surface with (b) $N=1\left(123^{\circ} \pm\right.$ $\left.0.5^{\circ}\right),(\mathrm{c}) N=2\left(128^{\circ} \pm 0.5^{\circ}\right)$, and (d) $N=4\left(145^{\circ} \pm 0.5^{\circ}\right)$.

measured by AFM) and is defined as the ratio of the textured surface area in contact with the liquid droplet and the flat surface before texturing [46].

To estimate the role of properties of blisters on the liquid contact area fraction $(f)$, we define the laser shot density per unit surface $(S)$ as $S=\frac{L}{v \cdot \Delta \mathrm{X}}$. Here, $L$ and $v$ together provide the total number of laser shots along the scan direction, and $\Delta \mathrm{X}$ in the orthogonal direction. The parameters $(L, v, \Delta \mathrm{X})$ for the laser-patterned surfaces of $N=1,2$, and 4 shots per focal spot are $(500 \mathrm{~Hz}, 3 \mathrm{~mm} / \mathrm{s}, 10 \mu \mathrm{m}),(500 \mathrm{~Hz}, 0.5 \mathrm{~mm} / \mathrm{s}$, $9 \mu \mathrm{m})$, and $(2 \mathrm{kHz}, 1 \mathrm{~mm} / \mathrm{s}, 5 \mu \mathrm{m})$, respectively. From this, we calculated the laser shot densities for the laser-patterned surfaces of $N=1,2$, and 4 shots/spot to be 0.017 shots $/ \mu \mathrm{m}^{2}$, 0.11 shots $/ \mu \mathrm{m}^{2}$, and 0.4 shots $/ \mu \mathrm{m}^{2}$, respectively. These calculations showed that the laser-patterned surface with $N=4$ shots/spot had the maximum laser shot density per unit surface $(S)$ and had the maximum contact angle $\left(145^{\circ}\right)$ shown in Fig. 4(d).

We note that the shape of the nanoscale structure of the blisters also plays a key role. Complex rough patterns such as triangular and fractal-like structures were shown to be superhydrophobic [47]. In the overlapped regions of the laser shots, we observed complex rough patterns involving substructures for each of these individual structures (details are in Supplemental Material Sec. VIII [26]). From AFM line scan profiles (shown in Supplemental Material Sec. VIII [26]), we found that the one-shot surface had smooth structures compared to two-shot and four-shot surfaces which had nanoscale substructures (fractal-like spikes on blisters). The presence of these substructures, caused by increasing the laser shot density per surface, plays a major role in minimizing the overall contact area fraction leading to increased contact angles.

Since the characterization of such complex surfaces involving multiscale roughness is difficult, we indirectly estimated the liquid contact area fractions $(f)$ from the experimental values of $\Theta_{0}$ and $\Theta_{\mathrm{CB}}$ (details are in Supplemental Material Sec. VII [26]) and indicated them in Fig. 4. This formula is valid for a surface that is flat and chemically homogeneous. The angle $\Theta_{0}$ (Young's angle) corresponds to the contact angle shown by a water droplet, which is $110^{\circ}$ for pristine surfaces of polyimide. Since we used the same pristine polyimide surface for laser-texturing experiments, we set $\Theta_{0}$ to be $110^{\circ}$ (as reference) and obtained the contact area fraction $(f)$ curve for various contact angles greater than $110^{\circ}$, owing to the fact that the addition of laser-induced surface texture increases contact angle of a surface. The calculated contact area fractions are mapped to the measured contact angles in Fig. 4.

Thus, the addition of the laser-induced surface texture in back illumination creates structures and substructures, minimizing the contact area fraction $(f)$. The smaller the contact area fraction, the higher the air pocket density that gives rise to the superhydrophobic behavior. Figure 4(b) shows a contact angle of $123^{\circ}\left( \pm 0.5^{\circ}\right)$ for a laser-patterned surface with $N=1$ at $290 \mathrm{~nJ}$ energy $\left(\sim 6.2 \mathrm{~J} / \mathrm{cm}^{2}\right)$. The increase in contact angle by $13^{\circ}$ is due to the increased surface texture caused by the formation of blisters. Since the film thickness was $1.3 \mu \mathrm{m}$, we lowered the energy to avoid rupturing of the film [13]. We decreased the energy to $260 \mathrm{~nJ}\left(\sim 5.6 \mathrm{~J} / \mathrm{cm}^{2}\right)$ for $N=2$ and $220 \mathrm{~nJ}\left(\sim 4.7 \mathrm{~J} / \mathrm{cm}^{2}\right)$ for $N=4$. These surfaces showed contact angles of $128^{\circ}\left( \pm 0.5^{\circ}\right)$ and $145^{\circ}\left( \pm 0.5^{\circ}\right)$ [Figs. $4(\mathrm{c})$ 
and 4(d)], due to creation of both structures and substructures. The same experiment could be carried out at lower energy, say $220 \mathrm{~nJ}\left(\sim 4.7 \mathrm{~J} / \mathrm{cm}^{2}\right)$, but in this case, the diameter of blisters is smaller. Thus, a smaller pitch would be required to overlap blisters.

These results clearly indicate that the increase in hydrophobicity in polymers when irradiated in back illumination is due to the creation of complex surfaces (involving substructures along with micro/nanostructures, similar to fractal-type structures in Ref. [47]). Although the surface roughness, as measured by an AFM, for the patterned surface of $N=1$ was higher than that of $N=2$, the density of air pockets $N=1$ was slightly lower than for $N=2$ due to a change in pitch. The pitch for $N=1$ and $N=2$ surfaces were $10 \mu \mathrm{m}$ and $9 \mu \mathrm{m}$, respectively. The higher the pitch, the lower the surface structural density and thereby the density of air pockets. For the patterned surface of $N=4$, the density of air pockets was even higher when the pitch was reduced to half $(\Delta \mathrm{X}=5 \mu \mathrm{m})$. These quantitative results clearly indicate that the adhesive properties of a polymer surface can be controlled by adding a texture in back illumination.

Based on our surface studies and related contact angle measurements, we propose a two-step LIFT procedure (considering the initial surface to be hydrophobic) to obtain a contaminant-free transfer of sensitive materials such as cells and organelles. In the first step, we would add only a texture to the surface in back illumination (preserving the surface chemistry) to minimize the surface adhesion. In the second step, we would irradiate the transformed surface with another laser pulse for a gentle desorption of the sensitive materials from the surface.

\section{CONCLUSIONS}

We studied ultrafast-laser-induced photochemical and morphological changes in polyimide thin films using FIB, XPS, AFM, and water contact angle measurements. Upon nonlinear interaction of ultrafast light with a polyimide film at the interface, the thin film was locally transformed and delaminated from the substrate, leading to formation of a blister and an embedded modified layer with a different phase contrast beneath the film. Both XPS and FIB measurements show that the chemical changes are confined to the glass-polyimide interface. The morphological change due to blister formation is evident through FIB measurements. Blister-patterned surfaces show increased contact angle of water droplets due to increased surface texture and therefore, increased hydrophobicity. By isolating morphological changes from chemical modification, we show that the addition of surface texture increases hydrophobicity and thereby decreases surface adhesion. We have demonstrated that back-illuminated surfaces preserve the surface chemistry and the resulting adhesion arises only from morphological changes.

The reduced adhesion that we have observed may be useful for contaminant-free LIFT since laser-induced chemical changes can be confined near the glass-polymer interface while morphological changes can help materials to gently desorb. In addition, confining the region of chemical change is essential for 3D optical data storage based on laser-induced fluorescence from polymers while isolated morphological changes may help create water repellent surfaces for applications in de-icing and defrosting.

\section{ACKNOWLEDGMENTS}

All authors acknowledge financial support from Natural Sciences and Engineering Research Council of Canada (NSERC) Engage (Grant No. EGP523138-18) and Discovery (Grant No. RGPIN-2019-04603), Ontario Centres of Excellence Voucher for innovation and Productivity I Program (Grant No. 29119), the Joint Centre for Extreme Photonics (JCEP) of the University of Ottawa and the National Research Council, and Fluidigm Canada, Markham, Ontario. Alan T. K. Godfrey acknowledges financial support from NSERC's Postgraduate Scholarship-Doctoral and University of Ottawa's Excellence Scholarship. We acknowledge Dr. Maohui Chen for training in atomic force microscopy, Dr. Choloong Hahn for FIB measurements, Tony Olivieri for training related to polyimide film fabrication, and Dr. Alexander Sander for acquiring XPS spectra. We acknowledge Dr. Zygmunt J. Jakubek for helping us to acquire absorption spectra for pristine and laser-modified polyimide (Supplemental Material [26]). We acknowledge the help received from Charbel Atallah, Ph.D. student, Department of Chemical and Biological Engineering, University of Ottawa for water drop contact angle measurements. We acknowledge help received from laboratory engineer Yu-Hsuan Wang.
[1] D. L. N. Kallepalli, A. M. Alshehri, D. T. Marquez, L. Andrzejewski, J. C. Scaiano, and R. Bhardwaj, Ultra-high density optical data storage in common transparent plastics, Sci. Rep. 6, 26163 (2016).

[2] D. L. N. Kallepalli, R. Kuladeep, S. V. Rao, and D. N. Rao, Luminescent microstructures in bulk and thin films of PMMA, PDMS, PVA, and PS fabricated using femtosecond direct writing technique, Chem. Phys. Lett. 503, 57 (2011).

[3] S. Nolte, M. Will, J. Burghoff, and A. Tuennermann, Femtosecond waveguide writing: A new avenue to three-dimensional integrated optics, Appl. Phys. A 77, 109 (2003).
[4] Y. Bellouard, A. A. Said, and P. Bado, Integrating optics and micro-mechanics in a single substrate: A step toward monolithic integration in fused silica, Opt. Express 13, 6635 (2005).

[5] N. T. Kattamis, P. E. Purnick, R. Weiss, and C. B. Arnold, Thick film laser induced forward transfer for deposition of thermally and mechanically sensitive materials, Appl. Phys. Lett. 91, 171120 (2007).

[6] M. S. Brown, N. T. Kattamis, and C. B. Arnold, Time-resolved study of polyimide absorption layers for blister-actuated laser-induced forward transfer, J. Appl. Phys. 107, 083103 (2010). 
[7] M. S. Brown, C. F. Brasz, Y. Ventikos, and C. B. Arnold, Impulsively actuated jets from thin liquid films for high-resolution printing applications, J. Fluid Mech. 709, 341 (2012).

[8] N. T. Kattamis, M. S. Brown, and C. B. Arnold, Finite element analysis of blister formation in laser-induced forward transfer, J. Mater. Res. 26, 2438 (2011).

[9] J. P. McDonald, V. R. Mistry, K. E. Ray, S. M. Yalisove, J. A. Nees, and N. R. Moody, Femtosecond-laser-induced delamination and blister formation in thermal oxide films on silicon (100), Appl. Phys. Lett. 88, 153121 (2006).

[10] J. R. Serranoand D. G. Cahill, Laser-induced blistering of thin $\mathrm{SiO}_{2}$ on $\mathrm{Si}$, Microscale Thermophys. Eng. 9, 155 (2005).

[11] N. T. Goodfriend, S. V. Starinskiy, O. A. Nerushev, N. M. Bulgakova, A. V. Bulgakov, and E. E. B. Campbell, Laser pulse duration dependence of blister formation on backradiated Ti thin films for BB-LIFT, Appl. Phys. A 122, 154 (2016).

[12] D. L. N. Kallepalli, A. T. K. Godfrey, J. Walia, F. Variola, A. Staudte, C. Zhang, Z. J. Jakubek, and P. B. Corkum, Multiphoton laser-induced confined chemical changes in polymer films, Opt. Express 28, 11267 (2020).

[13] A. T. K. Godfrey, D. L. N. Kallepalli, J. Ratté, C. Zhang, and P. B. Corkum, Femtosecond-Laser-Induced Nanoscale Blisters in Polyimide Thin Films through Nonlinear Absorption, Phys. Rev. Appl. 14, 044057 (2020).

[14] D. M. Rayner, A. Naumov, and P. B. Corkum, Ultrashort pulse non-linear optical absorption in transparent media, Opt. Express 13, 3208 (2005).

[15] M. Forster, W. Kautek, N. Faure, E. Audouard, and R. Stoian, Periodic nanoscale structures on polyimide surfaces generated by temporally tailored femtosecond laser pulses, Phys. Chem. Chem. Phys. 13, 4155 (2011).

[16] C. De Marco, S. M. Eaton, R. Suriano, S. Turri, M. Levi, R. Ramponi, G. Cerullo, and R. Osellame, Surface properties of femtosecond laser ablated PMMA, ACS Appl. Mater. Interfaces 2, 2377 (2010).

[17] C. Cheng, S. Wang, J. Wu, Y. Yu, R. Li, S. Eda, J. Chen, G. Feng, B. Lawrie, and A. Hu, Bisphenol a sensors on polyimide fabricated by laser direct writing for onsite river water monitoring at attomolar concentration, ACS Appl. Mater. Interfaces 8, 17784 (2016).

[18] M. Tang, Laser ablation of metal substrates for superhydrophobic effect, J. Laser Micro/Nanoeng. 6, 6 (2011).

[19] J. Long, P. Fan, D. Gong, D. Jiang, H. Zhang, L. Li, and M. Zhong, Superhydrophobic surfaces fabricated by femtosecond laser with tunable water adhesion: From lotus leaf to rose petal, ACS Appl. Mater. Interfaces 7, 9858 (2015).

[20] S. Moradi, S. Kamal, P. Englezos, and S. G. Hatzikiriakos, Femtosecond laser irradiation of metallic surfaces: Effects of laser parameters on superhydrophobicity, Nanotechnology $\mathbf{2 4}$, 415302 (2013).

[21] U. Hermens, S. V. Kirner, C. Emonts, P. Comanns, E. Skoulas, A. Mimidis, H. Mescheder, K. Winands, J. Krüger, E. Stratakis, and J. Bonse, Mimicking lizard-like surface structures upon ultrashort laser pulse irradiation of inorganic materials, Appl. Surf. Sci. 418, 499 (2017).

[22] B. E. J. Lee, H. Exir, A. Weck, and K. Grandfield, Characterization and evaluation of femtosecond laser-induced sub-micron periodic structures generated on titanium to improve osseointegration of implants, Appl. Surf. Sci. 441, 1034 (2018).
[23] M. Zuo, T. Takeichi, A. Matsumoto, and K. Tsutsumi, Surface characterization of polyimide films, Colloid Polym. Sci. 276, 555 (1998).

[24] Z. K. Wang, H. Y. Zheng, C. P. Lim, and Y. C. Lam, Polymer hydrophilicity and hydrophobicity induced by femtosecond laser direct irradiation, Appl. Phys. Lett. 95, 111110 (2009).

[25] A. M. Alshehri, S. Hadjiantoniou, R. J. Hickey, Z. Al-Rekabi, J. L. Harden, A. E. Pelling, and V. R. Bhardwaj, Selective cell adhesion on femtosecond laser-microstructured polydimethylsiloxane, Biomed. Mater. 11, 015014 (2016).

[26] See Supplemental Material at http://link.aps.org/supplemental/ 10.1103/PhysRevMaterials.5.045201 for the absorption spectrum of pristine polyimide in Fig. S1 indicated no linear absorption in the visible region from $450 \mathrm{~nm}$ to $800 \mathrm{~nm}$. Therefore, any absorption at $800 \mathrm{~nm}$ requires a minimum of 2 photons to be absorbed for modification.

[27] A. Kozbial, Z. Li, C. Conaway, R. McGinley, S. Dhingra, V. Vahdat, F. Zhou, B. D'Urso, H. Liu, and L. Li, Study on the surface energy of graphene by contact angle measurements, Langmuir 30, 8598 (2014).

[28] T.-S. Wong, S. H. Kang, S. K. Y. Tang, E. J. Smythe, B. D. Hatton, A. Grinthal, and J. Aizenberg, Bioinspired selfrepairing slippery surfaces with pressure-stable omniphobicity, Nature (London) 477, 443 (2011).

[29] W. Zheng, Surface wetting characteristics of rubbed polyimide thin films, in Polymer Thin Films, edited by A. A. Hashim (IntechOpen Limited, Rijeka, Croatia, 2010).

[30] R. Fardel, M. Nagel, F. Nüesch, T. Lippert, and A. Wokaun, Fabrication of organic light-emitting diode pixels by laserassisted forward transfer, Appl. Phys. Lett. 91, 061103 (2007).

[31] D. P. Banks, K. Kaur, R. Gazia, R. Fardel, M. Nagel, T. Lippert, and R. W. Eason, Triazene photopolymer dynamic release layer-assisted femtosecond laser-induced forward transfer with an active carrier substrate, Europhys. Lett. 83, 38003 (2008).

[32] P. Delaporte and A.-P. Alloncle, Laser-induced forward transfer: A high resolution additive manufacturing technology, Opt. Laser Technol. 78, 33 (2016).

[33] N. R. Schiele, D. T. Corr, Y. Huang, N. A. Raof, Y. Xie, and D. B. Chrisey, Laser-based direct-write techniques for cell printing, Biofabrication 2, 032001 (2010).

[34] C. B. Arnold, P. Serra, and A. Piqué, Laser direct-write techniques for printing of complex materials, MRS Bull. 32, 23 (2007).

[35] G. Jing, Y. Wang, T. Zhou, S. F. Perry, M. T. Grimes, and S. Tatic-Lucic, Cell patterning using molecular vapor deposition of self-assembled monolayers and lift-off technique, Acta Biomater. 7, 1094 (2011).

[36] M. Gruene, A. Deiwick, L. Koch, S. Schlie, C. Unger, N. Hofmann, I. Bernemann, B. Glasmacher, and B. Chichkov, Laser printing of stem cells for biofabrication of scaffold-free autologous grafts, Tissue Eng. Part C: Methods 17, 79 (2010).

[37] L. Koch, S. Kuhn, H. Sorg, M. Gruene, S. Schlie, R. Gaebel, B. Polchow, K. Reimers, S. Stoelting, N. Ma, P. M. Vogt, G. Steinhoff, and B. Chichkov, Laser printing of skin cells and human stem cells, Tissue Eng. Part C: Methods 16, 847 (2009).

[38] A. Palla-Papavlu, V. Dinca, C. Luculescu, J. Shaw-Stewart, M. Nagel, T. Lippert, and M. Dinescu, Laser induced forward transfer of soft materials, J. Opt. 12, 124014 (2010).

[39] P. Serra, M. Colina, J. M. Fernández-Pradas, L. Sevilla, and J. L. Morenza, Preparation of functional DNA microarrays 
through laser-induced forward transfer, Appl. Phys. Lett. 85, 1639 (2004).

[40] B. Hopp, T. Smausz, Z. Antal, N. Kresz, Z. Bor, and D. Chrisey, Absorbing film assisted laser induced forward transfer of fungi (Trichoderma conidia), J. Appl. Phys. 96, 3478 (2004).

[41] J. Barron, P. Wu, H. Ladouceur, and B. Ringeisen, Biological laser printing: A novel technique for creating heterogeneous 3-dimensional cell patterns, Biomed. Microdev. 6, 139 (2004).

[42] J. Xu, J. Liu, D. Cui, M. Gerhold, A. Y. Wang, M. Nagel, and T. K. Lippert, Laser-assisted forward transfer of multi-spectral nanocrystal quantum dot emitters, Nanotechnology 18, 025403 (2006).
[43] C. M. Chan and L.-T. Weng, Surface characterization of polymer blends by XPS and ToF-SIMS, Materials 9, 655 (2016).

[44] D. Zeng, K. Yung, and C. Xie, Xps investigation of the chemical characteristics of kapton films ablated by a pulsed TEA $\mathrm{CO}_{2}$ laser, Surf. Coat. Technol. 153, 210 (2002).

[45] http://www.casaxps.com/help_manual/manual_updates/ Basics_Quantification_of_XPS_Spectra.pdf.

[46] B. Wang, Y. Zhang, L. Shi, J. Li, and Z. Guo, Advances in the theory of superhydrophobic surfaces, J. Mater. Chem. 22, 20112 (2012).

[47] Q. Zheng and C. Lü, Size effects of surface roughness to superhydrophobicity, Proc. IUTAM 10, 462 (2014). 